\title{
Above, on, or shang (上)? Language and spatial representations among English-Mandarin bilinguals
}

\author{
Wei Xing Toh $^{1} \cdot$ Lidia Suárez ${ }^{2}$
}

Published online: 17 October 2017

(C) The Psychonomic Society, Inc. 2017

\begin{abstract}
This study investigated if exposure to spatial language could affect spatial cognition in EnglishMandarin bilinguals by focusing on contact/noncontact distinctions, an area that has been a source of contention in the language-and-thought literature. Sixty-three participants were first primed with sentences containing spatial terms (e.g., above, on) before performing a spatial decision task. Approximately half of the participants $(n=33)$ were primed in English; for the remaining participants ( $n$ $=30$ ), primes comprising Mandarin spatial terms-which mark spatial distinctions differently than in English (e.g., shang in Mandarin signifies both above and on in English)—were employed instead. Our findings revealed that participants' performance was influenced by spatial primes in the English experiment, thereby proffering evidence for thinking-for-speaking effects. However, these findings were not mirrored for the Mandarin experiment, confirming that the contact/noncontact specificity of spatial terms may have been instrumental in engendering the thinking-for-speaking effects observed in English.
\end{abstract}

Keywords Spatial cognition - Spatial language $\cdot$ Thinking for speaking

Wei Xing Toh

weixing.toh.2017@phdps.smu.edu.sg

1 School of Social Sciences, Singapore Management University, 90 Stamford Road, Level 4, Singapore 178903, Singapore

2 James Cook University, Singapore, Singapore
In the characterisation of space, there exists an assortment of words (e.g., left, right, over, below) that subserves fundamental human functions of navigation and communication. A still unresolved question within cognitive sciences is if spatial language modulates spatial representations. Traditionally, opinions on this topic have been discordant in which the idea that spatial language merely reflects spatial cognition (Li \& Gleitman, 2002; Munnich, Landau, \& Dosher, 2001) is contrasted with the viewpoint that spatial language strongly shapes spatial cognition (Levinson, 2003; Levinson, Kita, Haun, \& Rasch, 2002). A more recent approach is to consider spatial terms as tools providing concepts and strategies which, in turn, augment spatial representations (Gentner, 2003; Gentner \& Loewenstein, 2002). Consistent with this idea is the thinking-for-speaking hypothesis, whereby specific linguistic terms can highlight relevant features of our conceptual knowledge while ignoring or deemphasizing others (Athanasopoulos \& Bylund, 2013; Slobin, 1996).

A source of contention in extant language-and-thought literature concerns spatial terms that relate contact/noncontact distinctions. To illustrate, above, on, beneath, and below allow us to express varying levels of spatial distinctions between objects (Koh, 2013; Munnich et al., 2001). When conveying contact relations, the word $o n$ is used to refer to a figure object (e.g., bird) being supported by a reference object (e.g., tree). However, once contact breaks and the bird is in a superior position relative to the tree, the word above is used. Likewise, beneath denotes contact or near-contact associations and below implies relatively more distal relations. In contrast, other languages-like Japanese, Korean, and Mandarin-do not carve up space in the same manner as 
English. For instance, ue-ni (Japanese), wi-e (Korean), and shang (Mandarin) are commonly used to denote both above and on. ${ }^{1}$ Accordingly, such cross-linguistic differences may result in concomitant discontinuities in spatial cognition.

In this vein, Munnich et al. (2001) tested if English monolinguals would differ from Japanese and Korean monolinguals in spatial recognition memory. Participants were presented a series of pictures depicting various spatial relationships between figure and reference objects (e.g., a ball appeared to be either above or on the table). In every trial, participants had to view two pictures and discern if the second picture was identical to the first. It was found that English monolinguals performed comparably with Japanese and Korean monolinguals, which suggests the absence of language effects on contact/noncontact distinctions. However, in a recent adaptation of Munnich et al.'s (2001) experiment, Holmes, Moty, and Regier (2017) found that English monolinguals and Korean-English bilinguals who were early-English learners showed categorical sensitivity to contact/noncontact distinctions but not KoreanEnglish bilinguals who were late-English learners. ${ }^{2}$ Given these inconsistent findings, further empirical investigations are warranted.

Departing from existing research on contact/ noncontact relations, we tested for a more transient language-thought interaction-namely, thinking-forspeaking effects. Previous studies on thinking-forspeaking effects have demonstrated that language priming affects cognitive processes such as recognition memory and perception of motion (Feist \& Gentner, 2007; Kaschak et al., 2005; Loewenstein \& Gentner, 2005; Zwaan, Madden, Yaxley, \& Aveyard, 2004). To illustrate, in Feist and Gentner's (2007) study, participants read sentences (e.g., "The block is on the building") accompanied by 'standard' pictures which were borderline exemplars of the sentences (e.g., the block merely touching the building). After a 10-minute interval, in a yes/no recognition task where the earlierpresented 'standard' pictures had to be identified, participants made more false alarms for pictures which

\footnotetext{
${ }^{1}$ However, this is not to say that above and on cannot be differentiated in languages such as Mandarin and Korean. In Mandarin, shang can be modified to denote above (yi shang). In Korean, tte (meaning: floating) or putte (meaning: sticking) can be used to loosely denote above and on, respectively. It should still be noted that while these spatial distinctions are obligatorily marked in English, they are optional in other languages.

2 The spatial stimuli used in Holmes et al. (2017) were similar to those in Munnich et al. (2001). However, in Holmes et al.'s (2017) task, participants were shown four scenes simultaneously and had to differentiate a target scene from three identical distractor scenes by indicating the side of the screen (left or right) containing the target ("odd one out"). Categorical sensitivity for contact/ noncontact distinctions was signified by faster responses on between-category trials (e.g., target showing contact relation but distractors reflecting noncontact relations) than on within-category trials (e.g., target and distractors showing both contact relations).
}

were better exemplars of the spatial prepositions (e.g., the block fully resting on the building) than those that were poorer exemplars (e.g., the block hanging above the building), therefore alluding to the integral role of spatial prepositions in shaping spatial memory. Drawing upon such priming paradigms, we designed an experimental task to investigate the impact of contact/ noncontact spatial labels on spatial decisions. Further, we used an implicit dependent variable (i.e., click locations) to reduce the likelihood of language-congruent biases resulting from participants' conscious, strategic decisions (Winawer et al., 2007).

The outline of our experiment was as follows. In every trial, participants first read an English sentence (prime) embedded with spatial terms (e.g., above, on, beneath, below) and had to indicate if the sentence was logical (e.g., "The cat is on the table") or illogical (e.g., "The land is above the sky"). Subsequently, they had to click on a fixation point. Participants then attempted the spatial decision task which presented a figure comprising three rectangles: one narrower blue rectangle positioned in between two similar-sized grey and white rectangles. The blue rectangle remained fixed in the middle; when the grey rectangle was shown above the blue rectangle, the white rectangle was always below the blue rectangle, and vice versa. Participants had to click anywhere within the grey rectangle (see Fig. 1).

If spatial language influences spatial thinking, noncontact primes (above, below) would affect responses on the spatial decision task differently from contact primes (on, beneath). Specifically, even though participants were free to click anywhere within the grey rectangle, primes containing above would induce participants to click on higher positions with respect to the blue rectangle than would primes carrying on; similarly, primes comprising below would engender responses that are positioned lower from the blue rectangle than would primes involving beneath. Accordingly, this would proffer evidence for thinking-for-speaking effects, wherein language exposure influences spatial performance.

We further inquired about the locus of thinking-forspeaking effects by incorporating an additional experiment which was methodologically similar but the primes were presented in Mandarin, wherein the spatial terms, shang and xia, encompassed abovelon and beneath/ below, respectively. The Mandarin experiment served to disentangle two competing hypotheses behind language effects. A likely explanation is that such language effects were driven by the specificity of the spatial terms marking contact/noncontact distinctions. Alternatively, language effects could be engendered without spatial terms denoting contact/noncontact specificity. For instance, "The cup is on the table" can most realistically be 


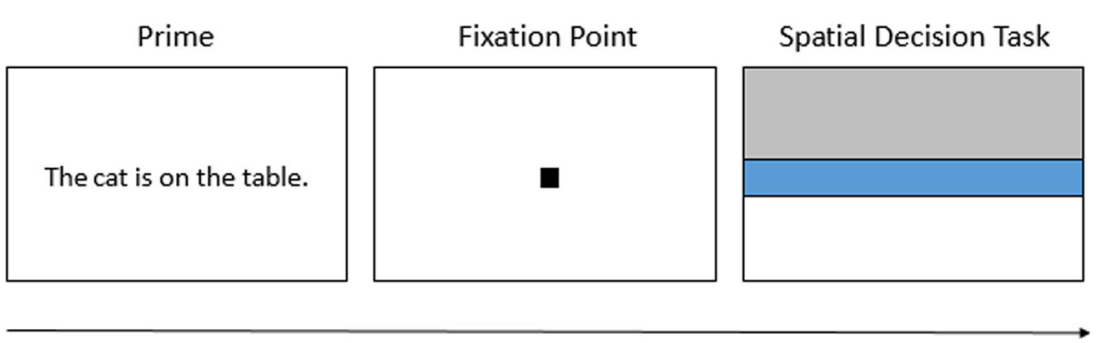

Fig. 1 The outline of an experimental trial.

understood in Mandarin (i.e., bei zi zai zhuo zi shang) as the cup having an upward, contact relation with the table, even if the word on is replaced by shang, a spatial term that does not overtly connote contact/noncontact distinctions. This highlights the potential role of contextual knowledge (i.e., how certain figure and reference objects are prototypically associated) in eliciting language effects. If thinking-for-speaking effects are dependent on the contact/noncontact specificity of spatial terms, then language effects would show up in the English, but not Mandarin, experiment. In contrast, if language effects appear in both experiments, then it would indicate a more general influence of contextual knowledge on thinking-for-speaking effects.

\section{Method}

\section{Participants}

Eighty-one English-Mandarin Singaporean bilinguals participated in the study for course credits or as volunteers. Eighteen participants ( 9 females) were assigned to the norming study to assess the comprehensibility of the primes. Sixty-three participants were allocated to either the English ( $n=33 ; 17$ females) or Mandarin $(n=30$; 20 females) experiment. Participants were comparable in language proficiency, frequency of use, age of exposure, and language dominance across the norming study, English experiment, and Mandarin experiment, $p \mathrm{~s}>$ .08 (see Appendix 1). Participants had normal or corrected-to-normal visual acuity without any other known language or developmental disorders.

\section{Materials}

Primes Each language experiment comprised 96 sentences (primes) embedded with spatial terms. In the English experiment, the spatial terms were above, on, beneath, and below; in the Mandarin experiment, the spatial terms were shang and $x_{i a}{ }^{3}$ The English and Mandarin sentences were translations of each other and conveyed an equal number of contact/noncontact relationships. The comprehensibility of the sentences was assessed in a norming study where participants evaluated if the sentences were logical or illogical. Sentences that failed to reach $85 \%$ accuracy were revised until the desired accuracy rate was obtained. In the final set of sentences used for the main experiments, the mean proportion of correctly identified sentences was $.89(S D=$ $.05)$. Half of the sentences were logical and the other half were illogical; illogical sentences were designed to ensure that the sentences were read meaningfully (see also Feist \& Gentner, 2007).

Spatial decision task A $18.9^{\circ} \times 15.2^{\circ}$ figure, which occupied most of the $31.6^{\circ} \times 25.4^{\circ}$ LCD screen, was presented. The figure comprised three rectangles: one $18.9^{\circ} \times 1.9^{\circ}$ blue rectangle, one $18.9^{\circ} \times 6.7^{\circ}$ white rectangle, and one $18.9^{\circ} \times 6.7^{\circ}$ grey rectangle.

To circumvent the possibility that participants might form an association between the primes and the spatial decision task, there was an equal proportion of matched and unmatched trials. An example of a matched trial is a prime carrying the preposition on followed by the spatial decision task showing a grey rectangle on top of the blue rectangle; matched trials were essential to assess the priming effects of language on spatial decisions. An instance of an unmatched trial is a prime containing the preposition above followed by the spatial decision task depicting a grey rectangle under the blue rectangle; unmatched trials were designed to disguise the relationship between the primes and the spatial decision task (see Fig. 2). All experimental manipulations (e.g., matched/unmatched, logical/illogical) were presented in a randomised order for each participant.

\footnotetext{
${ }^{3}$ The usage of on in our study was constrained to situations where the figure object was vertically aligned with, and in a superior position to, the reference object (e.g., "The cup is on the table"). We acknowledge that on in English and Mandarin can be flexibly used in other circumstances. For instance, in the sentence "The fly is on the wall", the figure object is horizontally aligned, and not vertically aligned, with the reference object. It would be worthwhile for future studies to explore how the polysemous nature of certain spatial terms, such as on, could affect language-thought interactions.
} 
Logical-Matched

Prime

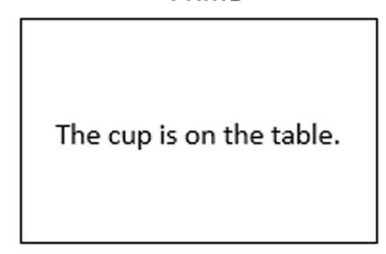

Spatial Decision Task

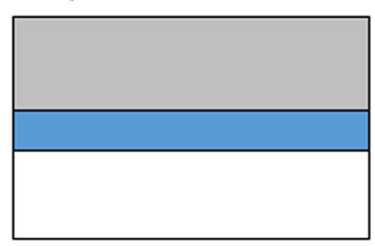

Logical-Unmatched

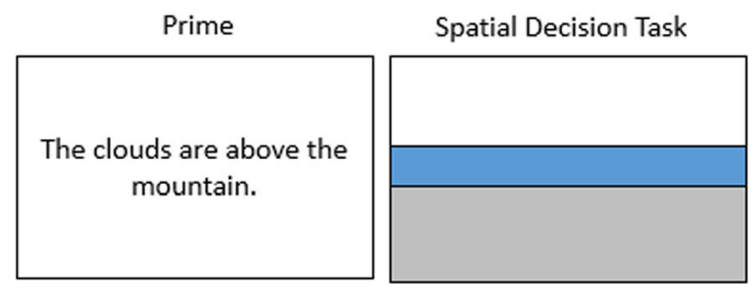

Fig. 2 Examples of matched (upper) and unmatched (lower) trials.

\section{Procedure}

All experimental stimuli were presented using E-Prime 2.0 software (Psychology Software Tools; http://www. pstnet.com) in a computer lab. After obtaining informed consent, participants were randomly assigned to either the English or Mandarin experiment. Instructions were rendered in the language of the experiment by the experimenter, who was a Singaporean English-Mandarin bilingual. The colour-response association phase was first initiated and participants were asked to figure out, via trial-and-error guessing, the correct response (i.e., click anywhere within the grey rectangle) to the spatial decision task. The purpose of this trial-and-error learning, instead of verbally instructing them to click within the grey rectangle, was to ensure that the responses on the spatial decision task were free from any explicit linguistic instructions; nonlinguistic tasks allow cleaner elucidation of language effects (e.g., Boroditsky, Fuhrman, \& McCormick, 2011). Figure 3 illustrates the colourassociation phase. After clicking on the fixation point, participants were shown the spatial decision task and were instructed to click anywhere on the screen. Onscreen feedback was given to indicate if their responses were correct (i.e., clicked within the grey rectangle) or incorrect (i.e., clicked anywhere else). To ensure that participants knew the correct response, 10 consecutive correct attempts were needed to terminate the colourresponse association phase.

Next, the priming phase was initiated (see Fig. 1). In each of the 96 trials, participants read a sentence and deduced its logicality by pressing $\mathrm{X}$ (Yes) or Z (No) keys on the keyboard. They then clicked on the fixation
Illogical-Matched

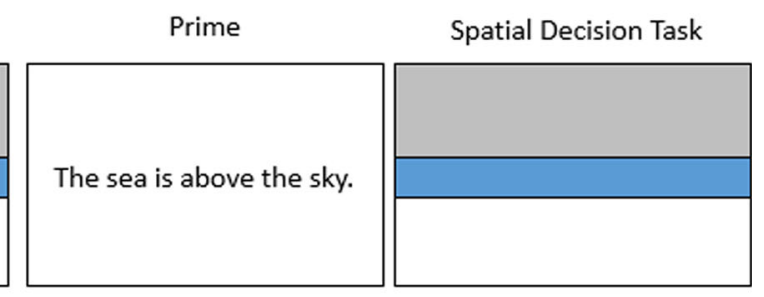

Illogical-Unmatched

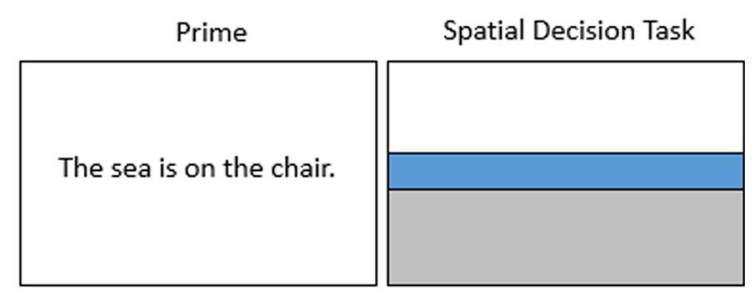

point and performed the spatial decision task in which they were instructed to respond similarly as they did in the colour-response association phase (i.e., to click within the grey rectangle). However, feedback on response accuracy was not provided this time (see Appendix 2 for experimental instructions). Lastly, participants completed a language background questionnaire, and were probed about the study's purpose and dependent variable, neither of which were accurately predicted by any participant.

\section{Results}

Only matched trials that fulfilled the following criteria were selected to allow cleaner analysis of priming effects: (a) sentences that were correctly identified (e.g., participants pressed Yes for logical sentences), and (b) correct responses for the spatial decision task (i.e., participants clicked within the grey rectangle). ${ }^{4}$ Nevertheless, we analysed the unmatched trials and, expectedly, no priming effects were found, $p$ s $>.61$ (see Table 3 of Appendix 3 and Table 5 of Appendix 4).

A 2(Language Experiment: English, Mandarin) X 2(Spatial Direction: upper [above, on], lower [below, beneath]) X 2(Spatial Distinction: contact [on, beneath], noncontact [above, below]) mixed analysis of variance

\footnotetext{
${ }^{4}$ In deducing sentential logicality, the proportion of correctly identified sentences was reasonably high $(M=.92, S D=.07)$, thereby indicating that the sentences were comprehensible and were likely to have been meaningfully processed. Further, the grey rectangle was correctly clicked for a noticeably high mean proportion of $.97(S D=.06)$, which implied that the participants were responding as expected in the spatial decision task.
} 


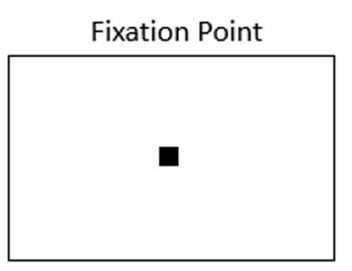

Fixation Point

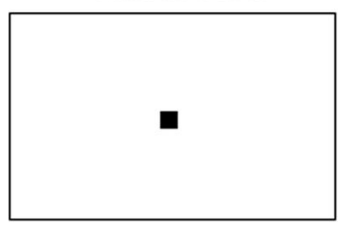

Spatial Decision Task

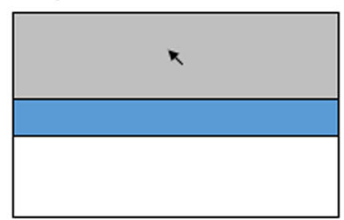

Spatial Decision Task

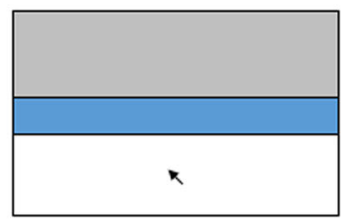

Feedback

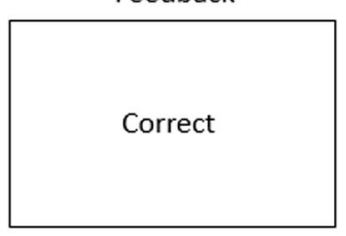

Feedback

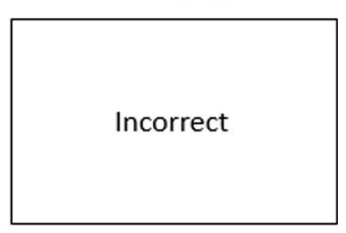

Fig. 3 Illustrations of correct (upper) and incorrect (lower) trials in the colour-response association phase.

(ANOVA) was performed by participants and by items. For the by-participant analysis, language experiment was a between-subject measure, while spatial direction and spatial distinction were within-subject measures. In the by-item analysis, language experiment was a withingroup variable and spatial direction and spatial distinction were between-group variables. The dependent variable was the location in which participants chose to click within the grey rectangle and was operationalised as the vertical distance (expressed in deg of visual angle) from the middle of the blue rectangle to participants' click locations (see Fig. 4). ${ }^{5}$

The ANOVA yielded a significant main effect of spatial direction $\left(F_{p}[1,61]=19.00, p<.001, \eta_{\mathrm{p}}{ }^{2}=.24\right.$; $\left.F_{i}[1,44]=39.25, p<.001, \eta_{\mathrm{p}}{ }^{2}=.47\right)$, indicating that participants clicked further from the blue object for the upper primes $\left(M_{p}=2.90, S D_{p}=0.65 ; M_{i}=2.89, S D_{i}=\right.$ $0.17)$ than for the lower primes $\left(M_{p}=2.68, S D_{p}=0.60\right.$; $\left.M_{i}=2.68, S D_{i}=0.17\right)$. The main effect of language experiment was significant only in the by-item analysis $\left(F_{i}[1,44]=5.43, p=.02, \eta_{\mathrm{p}}{ }^{2}=.11\right)$, signifying that responses in the English experiment $\left(M_{i}=2.82, S D_{i}=\right.$ 0.20 ) were generally more distant from the blue object than were responses in the Mandarin experiment $\left(M_{i}=\right.$ 2.75, $S D_{i}=0.20$ ).

Crucially, the main effect of spatial distinction was significant $\left(F_{p}[1,61]=6.50, p=.01, \eta_{\mathrm{p}}{ }^{2}=.10 ; F_{i}[1,44]=8.89, p=\right.$ $\left..01, \eta_{\mathrm{p}}{ }^{2}=.17\right)$, implying that noncontact primes $\left(M_{p}=2.83\right.$, $\left.S D_{p}=0.64 ; M_{i}=2.83, S D_{i}=0.17\right)$ engendered more distal responses from the blue object than did contact primes $\left(M_{p}=\right.$ 2.74, $\left.S D=0.58 ; M_{i}=2.73, S D_{i}=0.17\right)$, thereby constituting

\footnotetext{
5 The E-Prime program generated distances in pixel coordinates where 1 pixel was linearly equivalent to $0.05 \mathrm{~cm}$. The viewing distance was approximately $60 \mathrm{~cm}$.
}

evidence for the thinking-for-speaking hypothesis. Further, the language experiment $\mathrm{x}$ spatial distinction interaction was significant, $\left(F_{p}[1,61]=4.85, p=.03, \eta_{\mathrm{p}}{ }^{2}=.07 ; F_{i}[1,44]=\right.$ $\left.7.48, p=.01, \eta_{\mathrm{p}}{ }^{2}=.15\right)$, suggesting that contact/noncontact differences were asymmetric across the English and Mandarin experiments. Corroborating this finding, Bonferroni-corrected simple effects revealed that response differences between noncontact $\left(M_{p}=2.91, S D_{p}=0.53 ; M_{i}=2.83, S D_{i}=0.17\right)$ and contact primes $\left(M_{p}=2.74, S D_{p}=0.50 ; M_{i}=2.73, S D_{i}=0.17\right)$ were found in the English experiment $\left(F_{p}[1,61]=9.76, p=\right.$ $\left..008, \eta_{\mathrm{p}}{ }^{2}=.02 ; F_{i}[1,44]=25.46, p=.002, \eta_{\mathrm{p}}{ }^{2}=.37\right)$ but not the Mandarin experiment $\left(p s>.71, \eta_{\mathrm{p}}{ }^{2} \mathrm{~s}<.01\right.$; see Table 4 of Appendix 4 for all main effects and interactions).

In addition to the main analysis, we tested for response differences between above and on, as well as between below and beneath, in the English and Mandarin experiments. In line with our hypotheses, Bonferroni-corrected planned contrasts returned significant differences between above $\left(M_{p}=3.04\right.$, $\left.S D_{p}=0.61 ; M_{i}=3.03, S D_{i}=0.12\right)$ and on $\left(M_{p}=2.86, S D_{p}\right.$ $\left.=0.57 ; M_{i}=2.85, S D_{i}=0.16\right)$ responses $\left(t_{p}[32]=2.89, p=\right.$ $\left..03, d=0.51 ; t_{i}[22]=3.26, p=.02, d=1.33\right)$, as well as between below $\left(M_{p}=2.77, S D_{p}=0.52 ; M_{i}=2.78, S D_{i}=\right.$ $0.08)$ and beneath $\left(M_{p}=2.61, S D_{p}=0.51 ; M_{i}=2.61, S D_{i}=\right.$ $0.13)$ responses $\left(t_{p}[32]=2.74, p=.04, d=0.48 ; t_{i}[22]=4.05\right.$, $p=.002, d=1.65)$. However, these results were not mirrored for the Mandarin experiment, $p \mathrm{~s}>.99, d \mathrm{~s}<0.41$ (see Table 2 of Appendix 3). The asymmetric outcomes across the experiments attest to the contact/noncontact specificity of spatial terms, rather than contextual factors, in eliciting thinkingfor-speaking effects.

Lastly, we probed for the possibility of demand characteristics in the English experiment. For instance, priming effects could have amplified as the experiment progressed, signifying that participants were increasingly cognizant of the relation between the spatial terms and the spatial decision task. 

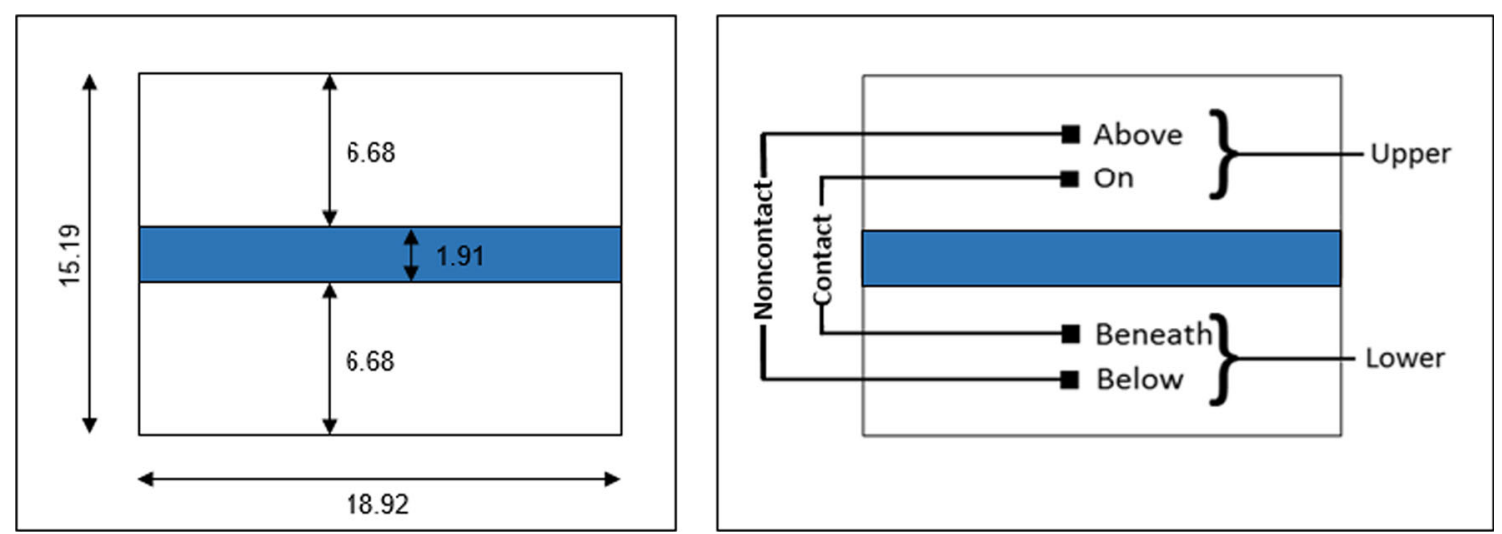

Fig. 4 Visualisations of figural dimensions (left) and experimental variables (right), spatial direction (upper, lower) and spatial distinction (noncontact, contact).

However, the size of priming effects ${ }^{6}$ was not significantly correlated with trial number (an index of experimental progress), $r=-.12, p=.26$, implying that priming effects did not grow larger across time. Another manifestation of demand characteristics is faster response times (RTs) on the spatial decision task for the matched, relative to the unmatched, trials, suggesting that participants might have formed an association between spatial primes and click locations. To this end, a byparticipant Wilcoxon signed-rank test showed that participants' RTs were comparable on the matched ( $M d n=489.10$ $\mathrm{ms}$ ) and unmatched ( $M d n=504.70 \mathrm{~ms})$ trials, $T=360.00, p=$ $.16, r=0.25$. $^{7}$ Together, these findings argue against the presence of demand characteristics.

\section{Discussion}

This study investigated if exposure to linguistic spatial primes could influence spatial decisions among EnglishMandarin bilinguals. Dovetailing previous reports of thinking-for-speaking effects (e.g., Feist \& Gentner, 2007; Zwaan et al., 2004), our results inform existing language-and-thought research by demonstrating thinking-for-speaking effects within the domain of contact/noncontact distinctions (Holmes et al., 2017; Munnich et al., 2001). Specifically, contact/noncontact differences in English spatial terms systematically swayed participants' decision to position their clicks in the spatial decision task. However, these effects were not observed when Mandarin spatial terms were used. This finding converges with previous studies (Athanasopoulos

\footnotetext{
${ }^{6}$ The size of priming effects was derived from the difference in mean click locations between noncontact (above, below) and contact (on, beneath) primes for each of the 96 trials.

${ }^{7}$ RTs which were 2.5 SDs above and below each participant's mean were removed. A nonparametric test was used as the Shapiro-Wilk test signified a violation of the assumption of normality, $W(32)=0.87, p<.001$.
}

et al., 2015; Holmes et al., 2017) underscoring bilinguals' conceptual flexibility, as shown by their sensitivity to divergent linguistic contexts.

Further, our results, highlighting that thinking-forspeaking effects are contingent on the specificity of spatial labels, can be accommodated by the 'language-augmented thought' hypothesis (Lupyan, 2012), which states that linguistic labels interact with conceptual structures by highlighting properties diagnostic of the relevant categories and, in the process, deepening intercategorical differences, making them more "fixed and distinct" (Lupyan, 2012, p. 265). In the context of our study, English prepositions, functioning as labels for conceptual categories, activate prototypical features of such spatial categories and momentarily sharpen categorical partitions between contact and noncontact representations; however, Mandarin spatial terms, which encode spatial relations dissimilarly, failed to elicit such categorical differentiation.

In summary, our findings add to the growing body of evidence suggesting that spatial language is intimately associated with spatial cognition. Notably, they coexist in a mutually reinforcing interdependence: Language does not merely express our thoughts, it appropriates readily available elements of our conceptual infrastructure, transiently making such linguistically congruent representations more salient than others.

\section{Appendix 1}


Table 1 Language profile of participants

\begin{tabular}{|c|c|c|c|c|c|c|}
\hline Variables & Norming Study $(n=18)$ & English Experiment $(n=33)$ & Mandarin Experiment $(n=30)$ & Overall $(N=81)$ & $F(2,78)$ & $p$ \\
\hline \multicolumn{7}{|l|}{ English Proficiency } \\
\hline Understanding & $5.78(0.81)$ & $6.10(0.88)$ & $5.97(0.89)$ & $5.98(0.87)$ & 0.76 & .47 \\
\hline Speaking & $5.89(0.76)$ & $6.03(0.81)$ & $5.90(1.03)$ & $5.95(0.88)$ & 0.23 & .80 \\
\hline Reading & $6.00(0.69)$ & $6.15(0.97)$ & $6.10(0.80)$ & $6.10(0.85)$ & 0.18 & .83 \\
\hline Writing & $5.89(0.76)$ & $5.94(0.90)$ & $5.87(0.97)$ & $5.90(0.89)$ & 0.05 & .95 \\
\hline \multicolumn{7}{|l|}{ Mandarin Proficiency } \\
\hline Understanding & $4.17(1.62)$ & $5.15(1.52)$ & $4.97(1.40)$ & $4.86(1.53)$ & 2.62 & .08 \\
\hline Speaking & $4.06(1.73)$ & $4.88(1.60)$ & $5.03(1.45)$ & $4.75(1.60)$ & 2.35 & .10 \\
\hline Reading & $3.67(1.75)$ & $4.24(1.80)$ & $4.33(1.63)$ & $4.15(1.73)$ & 0.92 & .40 \\
\hline Writing & $3.00(1.68)$ & $3.78(1.56)$ & $3.70(1.80)$ & $3.59(1.69)$ & 1.40 & .25 \\
\hline \multicolumn{7}{|c|}{ Frequency of English Use } \\
\hline Speaking & $5.00(0.00)$ & $4.97(0.17)$ & $5.00(0.00)$ & $4.99(0.11)$ & 0.72 & .49 \\
\hline Hearing & $5.00(0.00)$ & $5.00(0.00)$ & $5.00(0.00)$ & $5.00(0.00)$ & 0.00 & 1.0 \\
\hline Reading & $5.00(0.00)$ & $4.97(0.17)$ & $5.00(0.00)$ & $4.99(0.11)$ & 0.72 & .49 \\
\hline Writing & $5.00(0.00)$ & $4.94(0.24)$ & $4.97(0.18)$ & $4.96(0.19)$ & 0.60 & .55 \\
\hline \multicolumn{7}{|c|}{ Frequency of Mandarin Use } \\
\hline Speaking & $4.50(0.71)$ & $4.51(0.67)$ & $4.70(0.54)$ & $4.58(0.63)$ & 0.86 & .43 \\
\hline Hearing & $4.72(0.46)$ & $4.70(0.59)$ & $4.93(0.25)$ & $4.79(0.47)$ & 2.34 & .10 \\
\hline Reading & $3.72(1.13)$ & $3.94(0.79)$ & $3.80(0.96)$ & $3.84(0.93)$ & 0.36 & .70 \\
\hline Writing & $2.83(1.47)$ & $2.94(1.32)$ & $2.70(1.15)$ & $2.83(1.29)$ & 0.27 & .77 \\
\hline \multicolumn{7}{|l|}{ Age of Exposure (years) } \\
\hline English & $2.89(2.30)$ & $2.41(1.67)$ & $3.60(2.44)$ & $2.96(2.17)$ & 2.45 & .09 \\
\hline Mandarin & $3.78(3.14)$ & $3.03(2.21)$ & $3.27(2.64)$ & $3.29(2.58)$ & 0.48 & 62 \\
\hline \multicolumn{7}{|c|}{ Domain of Language Use (home) } \\
\hline English & 12 & 22 & 18 & 52 & - & - \\
\hline Mandarin & 6 & 11 & 12 & 29 & - & - \\
\hline \multicolumn{7}{|c|}{ Domain of Language Use (school/work) } \\
\hline English & 18 & 33 & 30 & 81 & - & - \\
\hline Mandarin & 0 & 0 & 0 & 0 & - & - \\
\hline \multicolumn{7}{|c|}{ Domain of Language Use (social) } \\
\hline English & 15 & 33 & 25 & 73 & - & - \\
\hline Mandarin & 3 & 0 & 5 & 8 & - & - \\
\hline \multicolumn{7}{|l|}{ Language Dominance } \\
\hline English-dominant & 11 & 22 & 18 & 51 & - & - \\
\hline Mandarin-dominant & 0 & 0 & 0 & 0 & - & - \\
\hline Balanced & 7 & 11 & 12 & 30 & - & - \\
\hline
\end{tabular}

Values for language proficiency (1: very few words, 7: native proficiency), frequency of language use (1: less than once a year, 2: every year, 3: every month, 4: every week, 5: every day), and age of exposure reflect means ( $+S D \mathrm{~s})$ of continuous variables. Values for domain of language use (English, Mandarin) and language dominance (English-dominant, Mandarin-dominant, balanced) denote frequency counts of categorical variables. Following Lim, Liow, Lincoln, Chan, and Onslow (2008), language dominance was determined using the following three criteria. For language proficiency scores, the dominant language was identified for two out of three dimensions: (a) difference in total scores between English and Mandarin >0; (b) difference in English and Mandarin scores for understanding, speaking, or reading modalities $>+1$ or $<-1$; and (c) difference in English and Mandarin scores for understanding, speaking, or writing modalities $>+1$ or $<-1$. For the frequency of language use, the dominant language had to be spoken and heard daily, and used for either reading or writing weekly. For the domain of language use, the dominant language had to be used the most in at least two out of the three contexts (i.e., domestic, formal, and social). The dominant language had to be consistently identified across the three criteria for participants to be classified as Mandarin- or English-dominant bilinguals; otherwise, participants would be categorised as balanced bilinguals. The proportions of Englishdominant and balanced bilinguals did not significantly differ across the norming study, English experiment, and Mandarin experiment, $\chi^{2}(2, N=81)=$ $0.33, p=.85$. 


\section{Appendix 2}

\section{Experimental instructions}

In the priming phase, participants were given the following instructions: "There will be 96 trials in total. In every trial, a sentence will first be shown. Two objects will be described in the sentence. As you are reading the sentence, try your best to think about the scenario portrayed in the sentence by visualising the spatial relationship between the two objects. Following which, indicate by pressing YES or NO if the scenario described in the sentence makes logical sense to you. An example of an illogical sentence is as follows: 'The ship is sailing on the clouds'. Try not to spend too much time on each sentence and interpret the sentence as simply as possible. Next, you will proceed with the spatial task which is identical to the colour-association task that you have attempted in the first phase of the experiment. However, feedback will not be provided this time. Nevertheless, please try to give as accurate a response as possible."

\section{Appendix 3}

Table 2 Mean distance $(+S D)$ of click locations in matched trials across English and Mandarin experiments

\begin{tabular}{|c|c|c|c|c|c|c|c|c|c|c|c|c|}
\hline \multirow[t]{2}{*}{ Experiment } & \multicolumn{6}{|l|}{ Upper } & \multicolumn{6}{|l|}{ Lower } \\
\hline & Above & On & $t$ & $d f$ & $p$ & $d$ & Below & Beneath & $t$ & $d f$ & $p$ & $d$ \\
\hline \multicolumn{13}{|c|}{ Analysis by Participants } \\
\hline English & $3.04(0.61)$ & $2.86(0.57)$ & 2.89 & 32 & .03 & 0.51 & $2.77(0.52)$ & $2.61(0.51)$ & 2.74 & 32 & .04 & 0.48 \\
\hline Mandarin & $2.84(0.82)$ & $2.86(0.71)$ & -0.23 & 29 & .99 & -0.04 & $2.68(0.76)$ & $2.64(0.69)$ & 0.74 & 29 & .99 & 0.12 \\
\hline \multicolumn{13}{|c|}{ Analysis by Items } \\
\hline English & $3.03(0.12)$ & $2.85(0.16)$ & 3.26 & 22 & .02 & 1.29 & $2.78(0.08)$ & $2.61(0.13)$ & 4.05 & 22 & .002 & 1.62 \\
\hline Mandarin & $2.82(0.16)$ & $2.86(0.18)$ & -0.54 & 22 & .99 & -0.24 & $2.70(0.21)$ & $2.62(0.18)$ & 0.99 & 22 & .99 & 0.41 \\
\hline
\end{tabular}

Lowest possible value $=0.95$, highest possible value $=7.63$; in boldface, $p<.05$ after Bonferroni correction .

Table 3 Mean distance $(+S D)$ of click locations in unmatched trials across English and Mandarin experiments

\begin{tabular}{|c|c|c|c|c|c|c|c|c|c|c|c|c|}
\hline \multirow[t]{2}{*}{ Experiment } & \multicolumn{6}{|l|}{ Upper } & \multicolumn{6}{|l|}{ Lower } \\
\hline & Above & On & $t$ & $d f$ & $p$ & $d$ & Below & Beneath & $t$ & $d f$ & $p$ & $d$ \\
\hline \multicolumn{13}{|c|}{ Analysis by Participants } \\
\hline English & $2.78(0.58)$ & $2.72(0.51)$ & 0.84 & 32 & .99 & 0.17 & $2.91(0.64)$ & $2.92(0.61)$ & -0.22 & 32 & .99 & -0.03 \\
\hline Mandarin & $2.67(0.71)$ & $2.67(0.65)$ & -0.06 & 29 & .99 & 0.00 & $2.80(0.71)$ & $2.77(0.77)$ & 0.38 & 29 & .99 & 0.07 \\
\hline \multicolumn{13}{|c|}{ Analysis by Items } \\
\hline English & $2.75(0.13)$ & $2.72(0.13)$ & 0.64 & 22 & .99 & 0.23 & $2.91(0.13)$ & $2.92(0.18)$ & -0.14 & 22 & .99 & -0.07 \\
\hline Mandarin & $2.66(0.19)$ & $2.68(0.15)$ & -0.30 & 22 & .99 & -0.12 & $2.78(0.15)$ & $2.77(0.12)$ & 0.17 & 22 & .99 & 0.07 \\
\hline
\end{tabular}

Lowest possible value $=0.95$, highest possible value $=7.63 ; p$ s reflect Bonferroni-corrected values 


\section{Appendix 4}

Table 4 Main effects and interaction effects for matched trials

\begin{tabular}{|c|c|c|c|c|}
\hline Variables & $F$ & MSE & $p$ & $\eta_{\mathrm{p}}^{2}$ \\
\hline \multicolumn{5}{|l|}{ Analysis by Participants $(n=63)$} \\
\hline \multicolumn{5}{|l|}{ Main effects } \\
\hline Language Experiment & 0.45 & 1.40 & .50 & .01 \\
\hline Spatial Direction & 19.00 & 0.17 & .00 & .24 \\
\hline Spatial Distinction & 6.50 & 0.08 & .01 & .10 \\
\hline \multicolumn{5}{|l|}{ Two-way interactions } \\
\hline Language Experiment $\times$ Spatial Direction & 0.45 & 0.17 & .50 & .01 \\
\hline Language Experiment $\times$ Spatial Distinction & 4.85 & 0.08 & .03 & .07 \\
\hline Spatial Direction $\times$ Spatial Distinction & 0.11 & 0.06 & .74 & .00 \\
\hline \multicolumn{5}{|l|}{ Three-way interaction } \\
\hline Language Experiment $\times$ Spatial Direction $\times$ Spatial Distinction & 0.48 & 0.06 & .49 & .01 \\
\hline \multicolumn{5}{|l|}{ Analysis by Items $(n=48)$} \\
\hline \multicolumn{5}{|l|}{ Main effects } \\
\hline Language Experiment & 5.43 & 0.02 & .02 & .11 \\
\hline Spatial Direction & 39.25 & 0.03 & .00 & .47 \\
\hline Spatial Distinction & 8.89 & 0.03 & .01 & .17 \\
\hline \multicolumn{5}{|l|}{ Two-way interactions } \\
\hline Language Experiment $\times$ Spatial Direction & 1.29 & 0.02 & .26 & .03 \\
\hline Language Experiment $\times$ Spatial Distinction & 7.48 & 0.02 & .01 & .15 \\
\hline Spatial Direction $\times$ Spatial Distinction & 0.59 & 0.03 & .45 & .01 \\
\hline \multicolumn{5}{|l|}{ Three-way interaction } \\
\hline Language Experiment $\times$ Spatial Direction $\times$ Spatial Distinction & 1.13 & 0.02 & .29 & .03 \\
\hline
\end{tabular}

In boldface, $p<.05$. Only 48 items from the matched trials were selected for analysis by items as the remaining 48 items belonged to the unmatched trials. 
Table 5 Main effects and interaction effects for unmatched trials

\begin{tabular}{|c|c|c|c|c|}
\hline Variables & $F$ & MSE & $p$ & $\eta_{\mathrm{p}}^{2}$ \\
\hline \multicolumn{5}{|l|}{ Analysis by Participants $(n=63)$} \\
\hline \multicolumn{5}{|l|}{ Main effects } \\
\hline Language Experiment & 0.50 & 28.32 & .48 & .01 \\
\hline Spatial Direction & 7.21 & 3.61 & .01 & .11 \\
\hline Spatial Distinction & 0.26 & 1.38 & .61 & .00 \\
\hline \multicolumn{5}{|l|}{ Two-way interactions } \\
\hline Language Experiment $\times$ Spatial Direction & 0.29 & 3.61 & .60 & .01 \\
\hline Language Experiment $\times$ Spatial Distinction & 0.01 & 1.38 & .91 & .00 \\
\hline Spatial Direction $\times$ Spatial Distinction & 0.08 & 1.17 & .78 & .00 \\
\hline \multicolumn{5}{|l|}{ Three-way interaction } \\
\hline Language Experiment $\times$ Spatial Direction $\times$ Spatial Distinction & 0.67 & 1.17 & .42 & .01 \\
\hline \multicolumn{5}{|l|}{ Analysis by Items $(n=48)$} \\
\hline \multicolumn{5}{|l|}{ Main effects } \\
\hline Language Experiment & 9.30 & 0.54 & .00 & .18 \\
\hline Spatial Direction & 24.22 & 0.42 & .00 & .36 \\
\hline Spatial Distinction & 0.01 & 0.42 & .91 & .00 \\
\hline \multicolumn{5}{|l|}{ Two-way interactions } \\
\hline Language Experiment $\times$ Spatial Direction & 1.42 & 0.54 & .24 & .03 \\
\hline Language Experiment $\times$ Spatial Distinction & 0.08 & 0.54 & .78 & .00 \\
\hline Spatial Direction $\times$ Spatial Distinction & 0.01 & 0.42 & .91 & .00 \\
\hline \multicolumn{5}{|l|}{ Three-way interaction } \\
\hline Language Experiment $\times$ Spatial Direction $\times$ Spatial Distinction & 0.31 & 0.54 & .58 & .01 \\
\hline
\end{tabular}

In boldface, $p<.05$. The main effect of spatial direction was significant, denoting response differences between the upper $\left(M_{p}=2.71, S D_{p}=0.59 ; M_{\mathrm{i}}=\right.$ $\left.2.70, S D_{i}=0.14\right)$ and lower $\left(M_{p}=2.85, S D_{p}=0.65 ; M_{i}=2.84, S D_{i}=0.14\right)$ groups of spatial primes. Additionally, the significant main effect of language experiment in the by-item analysis suggests that participants were clicking further away from the blue object in the English experiment $\left(M_{i}=2.82, S D_{i}=\right.$ $0.15)$ than in the Mandarin experiment $\left(M_{i}=2.72, S D_{i}=0.16\right)$. Importantly, both the main effect of spatial distinction and the language experiment $\mathrm{x}$ spatial distinction interaction, which signify priming effects, were nonsignificant, $p \mathrm{~s}>.61$.

\section{References}

Athanasopoulos, P., \& Bylund, E. (2013). The "thinking" in thinking-forspeaking: Where is it? Language, Interaction and Acquisition, 4, 91-100. doi:https://doi.org/10.1075/lia.4.1.05ath

Athanasopoulos, P., Bylund, E., Montero-Melis, G., Damjanovic, L., Schartner, A., Kibbe, A., ... Thierry, G. (2015). Two languages, two minds: Flexible cognitive processing driven by language of operation. Psychological Science, 26, 518-526. doi:https://doi.org/ 10.1177/0956797614567509

Boroditsky, L., Fuhrman, O., \& McCormick, K. (2011). Do English and Mandarin speakers think about time differently?. Cognition, 118, 123-129. doi:https://doi.org/10.1016/j.cognition.2010.09.010

Feist, M. I., \& Gentner, D., (2007). Spatial language influences memory for spatial scenes. Memory \& Cognition, 35, 283-296. doi:https:// doi.org/10.3758/BF03193449

Gentner, D. (2003). Why we're so smart. In D. Gentner \& S. GoldinMeadow (Eds.), Language in mind: Advances in the study of language and thought (pp. 195-235). Cambridge, MA: MIT Press.

Gentner, D., \& Loewenstein, J. (2002). Relational language and relational thought. In E. Amsel \& J. P. Byrnes. Language, literacy, and cognitive development: The development and consequences of symbolic communication (pp. 87-120). Mahwah, NJ: Erlbaum.

Holmes, K. J., Moty, K., \& Regier, T. (2017). Revisiting the role of language in spatial cognition: Categorical perception of spatial relations in English and Korean speakers. Psychonomic Bulletin \& Review. Advance online publication. doi:https://doi.org/10.3758/ s13423-017-1268-x

Kaschak, M. P., Madden, C. J., Therriault, D. J., Yaxley, R. H., Aveyard, M., Blanchard, A. A., \& Zwaan, R. A. (2005). Perception of motion affects language processing. Cognition, 94, B79-B89. doi:https:// doi.org/10.1016/j.cognition.2004.06.005

Koh, A. B. B. (2013). The effect of bilingualism on the perception of distance (Unpublished undergraduate dissertation). James Cook University, Singapore.

Levinson, S. C. (2003). Space in language and cognition: Explorations in cognitive diversity. Cambridge, UK: Cambridge University Press.

Levinson, S. C., Kita, S., Haun, D. B., \& Rasch, B. H. (2002). Returning the tables: Language affects spatial reasoning. Cognition, 84, 155-188. doi:https://doi.org/10.1016/S0010-0277(02)00045-8

Li, P., \& Gleitman, L. (2002). Turning the tables: Language and spatial reasoning. Cognition, 83, 265-294. doi:https://doi.org/10.1016/ S0010-0277(02)00009-4

Lim, V. P., Liow, S. J. R., Lincoln, M., Chan, Y. H., \& Onslow, M. (2008). Determining language dominance in English-Mandarin bilinguals: Development of a self-report classification tool for clinical use. Applied Psycholinguistics, 29, 389-412. doi:https://doi.org/10. 1017/S0142716408080181

Loewenstein, J., \& Gentner, D. (2005). Relational language and the development of relational mapping. Cognitive Psychology, 50, 315-353. doi:https://doi.org/10.1016/j.cogpsych.2004.09.004 
Lupyan, G. (2012). What do words do? Toward a theory of languageaugmented thought. In B. H. Ross (Ed.), The psychology of learning and motivation (Vol. 57, pp. 255-297). Waltham, MA: Academic Press.

Munnich, E., Landau, B., \& Dosher, B. A. (2001). Spatial language and spatial representation: A cross-linguistic comparison. Cognition, 81, 171-208. doi:https://doi.org/10.1016/S0010-0277(01)00127-5

Slobin, D. I. (1996). From "thought and language" to "thinking for speaking" In J. J. Gumperz \& S. C. Levinson (Eds.), Rethinking linguistic relativity (pp. 70-96), New York, NY: Cambridge University Press.
Winawer, J., Witthoft, N., Frank, M. C., Wu, L., Wade, A. R., \& Boroditsky, L. (2007). Russian blues reveal effects of language on color discrimination. Proceedings of the National Academy of Sciences, 104, 7780-7785. doi:https://doi.org/10.1073/pnas. 0701644104

Zwaan, R. A., Madden, C. J., Yaxley, R. H., \& Aveyard, M. E. (2004). Moving words: Dynamic representations in language comprehension. Cognitive Science, 28, 611-619. doi:https://doi.org/10.1016/j. cogsci.2004.03.004 